\title{
Effect of Crystallographic Orientation on Structural Response of Silicon to Femtosecond Laser Irradiation
}

\section{Xin Zhang}

Beijing University of Technology

Liang Zhang

Southern University of Science and Technology

\section{Sergey Mironov}

Beijing University of Technology

\section{Rongshi Xiao}

Beijing University of Technology

\section{Liang Guo}

Southern University of Science and Technology

\section{Ting Huang ( $\square$ huangting@bjut.edu.cn )}

Beijing University of Technology https://orcid.org/0000-0001-7139-0614

\section{Regular papers}

Keywords: femtosecond laser, crystallographic orientation, amorphization, silicon.

Posted Date: February 4th, 2021

DOl: https://doi.org/10.21203/rs.3.rs-193694/v1

License: (c) (1) This work is licensed under a Creative Commons Attribution 4.0 International License.

Read Full License

Version of Record: A version of this preprint was published at Applied Physics A on February 21st, 2021. See the published version at https://doi.org/10.1007/s00339-021-04341-y. 


\title{
Effect of Crystallographic Orientation on Structural Response of Silicon to Femtosecond Laser Irradiation
}

\author{
Xin Zhang ${ }^{1}$, Liang Zhang ${ }^{2}$, Sergey Mironov ${ }^{1}$, Rongshi Xiao ${ }^{1}$, Liang Guo $^{2 *}$, and Ting Huang ${ }^{1 *}$ \\ ${ }^{1}$ High-Power and Ultrafast Laser Manufacturing Lab, Faculty of Materials and Manufacturing, \\ Beijing University of Technology, Beijing 100124, P. R. China \\ ${ }^{2}$ Department of Mechanical and Energy Engineering, Southern University of Science and \\ Technology, Shenzhen, Guangdong 518055, P. R. China \\ *Corresponding author: huangting@bjut.edu.cn; guol3@sustc.edu.cn
}

\begin{abstract}
Femtosecond laser has been widely utilized for modification of crystal structure to achieve desired functions. So far, however, the effect of crystallographic orientation on the induced structure by femtosecond laser processing has yet been comprehensively studied. The present work is undertaken in an attempt to fill this gap in our knowledge. To this end, commercial-purity $\mathrm{Si}$ is used as a target material and high-resolution transmission electron microscopy as well as electron backscatter diffraction are applied to examine the irradiation-induced microstructural changes. The structural response of the pulsed material is found to be principally influenced by the crystallographic orientation of the target surface. Specifically, at the surface orientation close to $\{111\}$, a pronounced amorphization effect is observed whereas no disordered material is detected at the orientations close to $\{100\}$. This phenomenon could be explained by the lowest crystallization speed required by the (111) surface due to its smallest surface energy. Compared with nanosecond laser, non-thermal melting induced by femtosecond laser induces mild thermal gradient and favors recrystallization.
\end{abstract}

Keywords: femtosecond laser; crystallographic orientation; amorphization; silicon.

\section{Introduction}

Femtosecond laser has been widely applied for crystal structure modification due to its merits such as high instantaneous intensity and short pulse duration, which could lead to nonlinear absorption and small heat-affected zone. Diverse works in this area include amorphization of crystalline $\mathrm{Si}$ [1-3], control of manganite electronic phase [4], ultrafast phase transition in $\mathrm{VO}_{2}$ [5], ultrafast structural transformation in $\mathrm{Ge}_{2} \mathrm{Sb}_{2} \mathrm{Te}_{5}$-based phase-change materials [6], and so on. Despite these achievements on various materials, these studies usually focused on the effect of femtosecond laser parameters such as fluence and wavelength, while the effect of the sample crystallographic orientation, especially for single crystal samples, has not attracted sufficient attention. Recently, it was found that crystallographic orientation could affect the formation of conical structure on $\mathrm{Si}$ and $\mathrm{Ge}$ as well as periodic surface structures on $\mathrm{Ni}$ during femtosecond laser processing $[7,8]$. Therefore, crystallographic orientation could also be a notable parameter in laser processing. Actually, there are a few early works discussing crystallographic orientation dependence of crystal growth or amorphization of Si heated by picosecond ( $>10 \mathrm{ps}$ ) or nanosecond laser [9-11]. However, since the pulse width of femtosecond laser is shorter than the characteristic timescale of 
carrier-lattice coupling (typically on the order of 1-10 ps), strong thermal non-equilibrium between carriers and lattice can be induced by femtosecond laser heating [12-14], which is fundamentally different from heating by nanosecond laser. Therefore, the structural response with femtosecond laser irradiation deserves specific investigation.

The present work attempts to shed more light on this issue. Femtosecond laser-induced amorphization was performed on surfaces of Si with different crystallographic orientations. Nearsurface morphology was characterized by conventional scanning electron microscopy (SEM), highresolution transmission electron microscopy (TEM), electron backscatter diffraction (EBSD) analysis and atomic force microscopy (AFM). The characterization results reveal that surfaces of different crystallographic orientations correspond to different tendencies to form amorphization and the (111) surface of Si favors amorphization compared with the other crystallographic planes.

\section{Material and methods}

The samples used in the present investigation were commercial polycrystalline Si and singlecrystalline Si wafers. A femtosecond laser (TRUMICRO-5000) with $1030 \mathrm{~nm}$ wavelength, $400 \mathrm{kHz}$ repetition rate, and 800 fs pulse width was used to irradiate the samples. The maximum single-pulse energy was $200 \mu \mathrm{J}$. A scanning galvanometer was used to control the laser beam scanning with a scanning rate of $2500 \mathrm{~mm} / \mathrm{s}$ and scanning intervals of $140 \mu \mathrm{m}$ to create irradiation spots in array. The surface was scanned by the laser beam with track displacement of $150 \mu \mathrm{m}$ to achieve area irradiation. For single-crystalline Si experiment, the focused beam diameter was $120 \mu \mathrm{m}$ using a ftheta optics with focal-length $f=420 \mathrm{~mm}$. For polycrystalline $\mathrm{Si}$ experiment, the focused beam diameter was $30 \mu \mathrm{m}$ using a f-theta optics with focal-length $f=100 \mathrm{~mm}$, and the beam diameter of $152 \mu \mathrm{m}$ was used by defocusing $3 \mathrm{~mm}$. The laser irradiation at different laser fluences from 0.29 $\mathrm{J} / \mathrm{cm}^{2}$ to $1.43 \mathrm{~J} / \mathrm{cm}^{2}$ was carried out in air. Laser light intensity was of Gaussian distribution.

SEM and EBSD examinations were conducted with a Hitachi S-4300SE field emission gun scanning electron microscope equipped with a TSL OIM ${ }^{\mathrm{TM}}$ EBSD system and operated at accelerating voltage of $25 \mathrm{kV}$. In EBSD, samples were tilted by 70 degrees for data collection. Crystallographic orientation data were collected directly from surfaces irradiated by single pulses, without additional polishing. Depending on purposes, different scan step sizes were used for mapping. Specifically, low-resolution (overview) EBSD maps were acquired using a scan step size of $5 \mu \mathrm{m}$, whereas higher-resolution maps were obtained with a step size of either 1 or $0.2 \mu \mathrm{m}$. On each diffraction pattern, seven Kikuchi bands were used for indexing to minimize the possibility of misindexing error. For TEM, microstructural specimens were obtained by using focused ion beam (FIB) milling in a FEI Helios instrument. A layer with carbon and Pt was coated on the surface of the specimens to protect the TEM sample during FIB. The microstructure observation was performed using a transmission electron microscope (ZEISS LIBRA-200FE), which was operated at acceleration voltage of $200 \mathrm{kV}$. The surface profile was examined using an atomic force microscope (BRUKER MultiMode 8).

\section{Results and discussion}

A typical SEM image, an EBSD Kikuchi-band-contrast map, and an EBSD crystallographic orientation map of the laser-irradiated spots on polycrystalline Si are shown in Figs. 1(a)-(c). The Kikuchi-band-contrast map is used as a quantitative measure of crystal disordering whereas the crystallographic orientation map shows the orientation of each grain. Of particular interest is the variation of morphology of the laser-induced spots from grain to grain. While those appear similar 
in different grains in the SEM image as illustrated in Fig. 1(a) ${ }^{1}$, EBSD measurements demonstrate essential sensitivity of the microstructures induced by femtosecond laser irradiation to the crystallographic orientation as illustrated in Figs. 1(b) and (c). Specifically, the (111) surface (blue regions in Fig. 1(c)) exhibits relatively dark-contrast spots as shown in Fig. 1(b). To provide a closer inspection of this phenomenon, a magnified image of a typical spot is given as an inset in the top right corner of Fig. 1(b). Within this spot, two microstructural zones are found, i.e., the relatively dark outer region and the brighter inner one. Throughout the manuscript, these two are defined as Zone D and C, respectively. As discussed below, the dark contrast is a result of misindexing of disordered material. In comparison, the near-(100) surfaces (i.e., the red-, pink-, orange- and even ultraviolet areas in Fig. 1(c)) shows no such features.

The above effect is observed for the entire range of the imposed laser fluences in this work, thus reflecting anisotropy of structural response to femtosecond laser irradiation. In order to provide a deeper insight into this issue, two single-crystalline Si wafers, with (111) and (100) surfaces respectively, were selected for more detailed investigation.

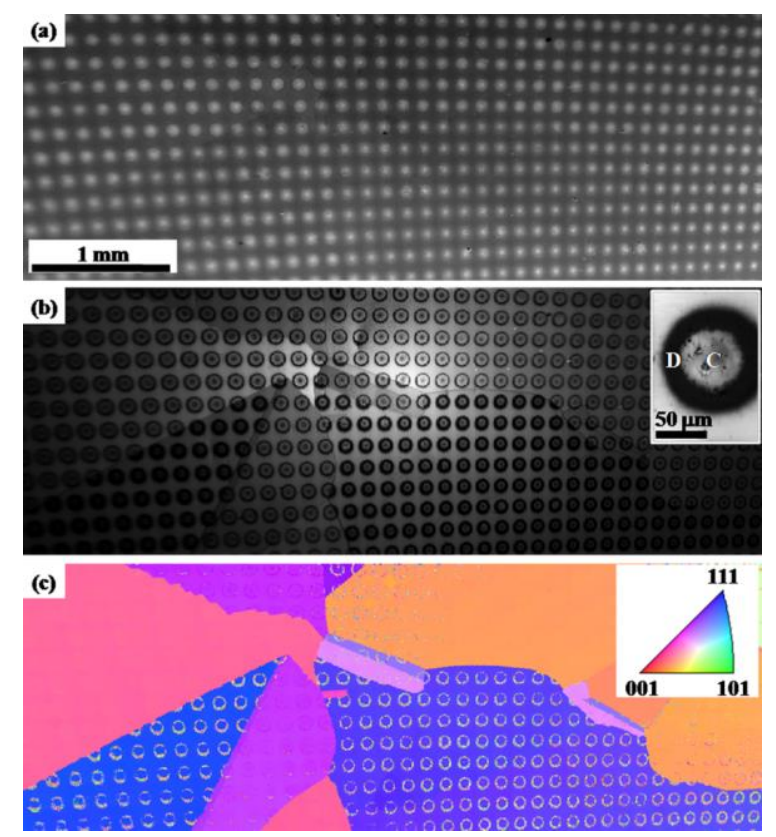

FIG. 1. SEM image (a), EBSD Kikuchi-band-contrast map (b), and EBSD crystallographic orientation map (c) showing anisotropic structural response to femtosecond laser irradiation. In (b), the inset is the magnified view of the laser-induced spot in the (111) surface. In (c), individual grains are colored according to their crystallographic orientation and the color code triangle is shown in the top right corner. Note: The data are taken from polycrystalline Si irradiated at laser peak-fluence of $0.99 \mathrm{~J} / \mathrm{cm}^{2}$.

SEM images and EBSD maps illustrating microstructural behavior of the single-crystalline sample with the (111) surface are summarized in Fig. 2. The outer parts of the irradiated spots on such-oriented surfaces are characterized by relatively dark color under SEM. Moreover, EBSD fails in indexing the crystallographic orientation in such areas as shown in Fig. 2(a)-(c). Both these

\footnotetext{
${ }^{1}$ Some inconsistency in spacing between the laser-irradiated spots seen in Fig. 1 is likely due to the perspective effect associated with the sample tilting in the SEM.
} 
characteristics indicate a high degree of crystal disordering. With higher laser fluence, the central section of the spots appears brighter and the crystallographic orientation there becomes welldetermined in EBSD mapping as shown in Figs. 2(b) and (c). The crystallographic orientation of the central part is found to be same as that of the unirradiated region. In addition, the disordered area produced by high laser fluences tends to shrink towards the spot periphery thus forming the ring-type feature. To provide a further insight into the microstructure distribution within the irradiated spots, high-resolution TEM was employed. Direct TEM observation reveals formation of amorphous layer in zone D of the laser irradiated surface (Fig. 2(d), c-Si abbreviates crystalline Si and the arrow shows a native oxide layer). On the other hand, no amorphous phase is found in zone $\mathrm{C}$ (not shown). The crystal disordering found in zone $\mathrm{D}$ well explains for the relatively dark contrast as well as the failure of EBSD in indexing this region. In addition, the TEM study of zone D also reveals a transition layer between the top amorphous layer and the bottom layer with a crystal structure, as demonstrated in Fig. 2(e). In the transition layer, the initial crystalline atomic arrangements of $\mathrm{Si}$ are transformed into a short-range order of amorphous $\mathrm{Si}$. In order to provide additional insight into microstructural behavior during laser pulsing, AFM image of the transition region between Zone $\mathrm{C}$ and Zone $\mathrm{D}$ is captured, and the radial surface profile of the same region is given in Fig. 2(f), which shows that Zone $\mathrm{C}$ is lower than Zone D, indicating apparent material removal in Zone $\mathrm{C}$ for the (111) surface.
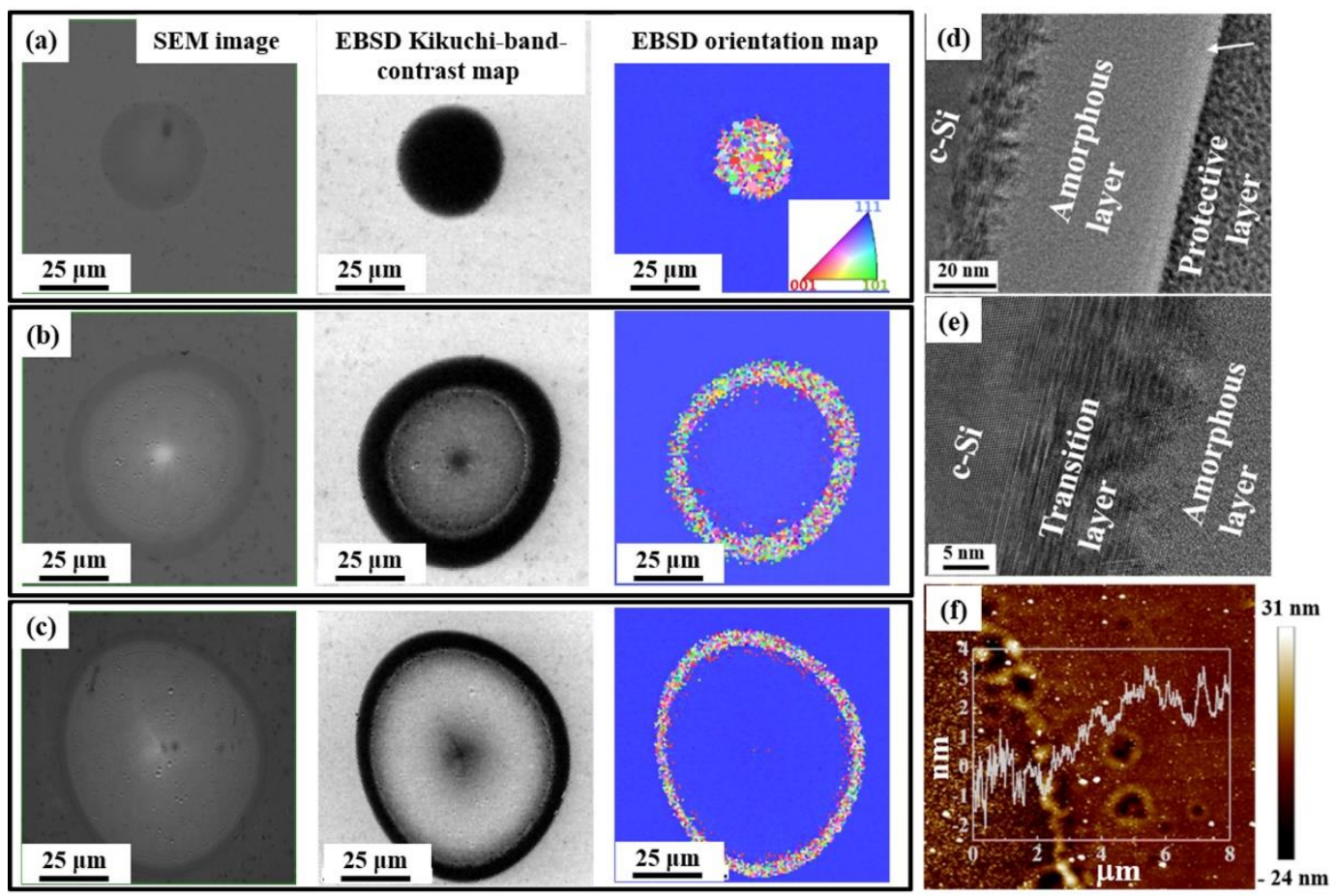

FIG. 2. SEM images and EBSD maps showing structural response of single-crystalline $\mathrm{Si}$ with the (111) surface to femtosecond laser peak-fluence of $0.29 \mathrm{~J} / \mathrm{cm}^{2}$ (a), $0.57 \mathrm{~J} / \mathrm{cm}^{2}$ (b), and $1.43 \mathrm{~J} / \mathrm{cm}^{2}$ (c). TEM observation of the microstructure $\left(0.57 \mathrm{~J} / \mathrm{cm}^{2}\right)$ in the amorphous-crystalline transition layer (zone D): low magnification (d) and high magnification (e). AFM image of the transition region between Zone $\mathrm{C}$ and Zone $\mathrm{D}$, and the radial surface profile of the same region $\left(0.57 \mathrm{~J} / \mathrm{cm}^{2}\right)$ (f). Note: in all the EBSD crystallographic orientation maps, regions are colored according to their crystallographic orientations and the color code triangle is given in the bottom right corner of (a). 
Some of the early experiments on femtosecond laser-induced modification on Si surface also distinguished regions of amorphization and crystallization [15-17], similar to zone $\mathrm{D}$ and zone $\mathrm{C}$ in this work for the (111) surface. The relationship between the laser-modified area and the corresponding laser pulse fluence is obtained and plotted in Fig. 3, from which the threshold fluence for ablation and amorphization is determined. The relation between the diameter of the damaged spot and the laser fluence is fitted according to $D^{2}=2 \omega_{0}^{2} \ln \left(F_{0} / F_{\text {th }}\right)$, where $D$ is the diameter of the ring-type feature (the inner diameter for ablation threshold and the outer diameter for amorphization threshold), $F_{0}$ is the femtosecond laser peak-fluence, and $\omega_{0}$ is the $e^{-2}$ radius $(60 \mu \mathrm{m})$ for a spatially Gaussian beam $[9,18]$. Based on the results for $(111)$ surface, the threshold fluence $\left(F_{\text {th }}\right)$ for ablation and amorphization are $0.45 \mathrm{~J} / \mathrm{cm}^{2}$ and $0.28 \mathrm{~J} / \mathrm{cm}^{2}$, respectively as shown in Fig. 3. Due to the spatial characteristic of the Gaussian beam, the intensity over the central part of the beam exceeds the ablation threshold and the intensity around the edges of the beam only exceeds the amorphization threshold, as shown in Figs. 2(b) and (c).

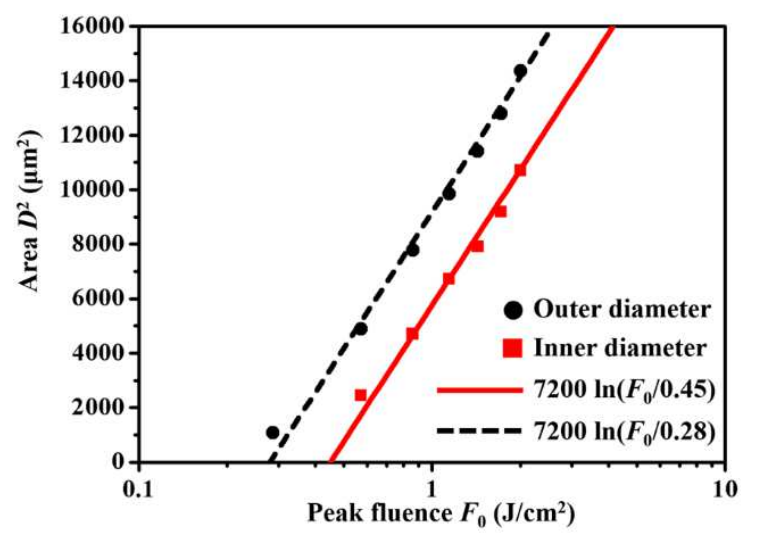

FIG. 3. Dependence of ablation area (Zone C) and amorphization area (Zone D) on laser peakfluence. Note: The data were taken from single-crystalline $\mathrm{Si}$ with the (111) surface.

Structural response of the single-crystalline sample with the (100) surface is distinctly different from that discussed above. Specifically, while SEM observation of the laser-induced spots reveals a notable contrast, EBSD measurements show only minor difference in microstructure as well as negligible difference in crystallographic orientation compared with those of the unaffected region as shown in Figs. 4(a)-(c). TEM observation reveals only a native oxide layer at the laser irradiated surface (marked by arrows in Figs. 4 (d) and (e)) but no signs of amorphous Si. In the AFM image of the transition region between Zone C and Zone D (Fig. 4(f)), the radial surface profile exhibits a similar pattern as that of the (111) surface, i.e. Zone $\mathrm{C}$ is lower than Zone D. 

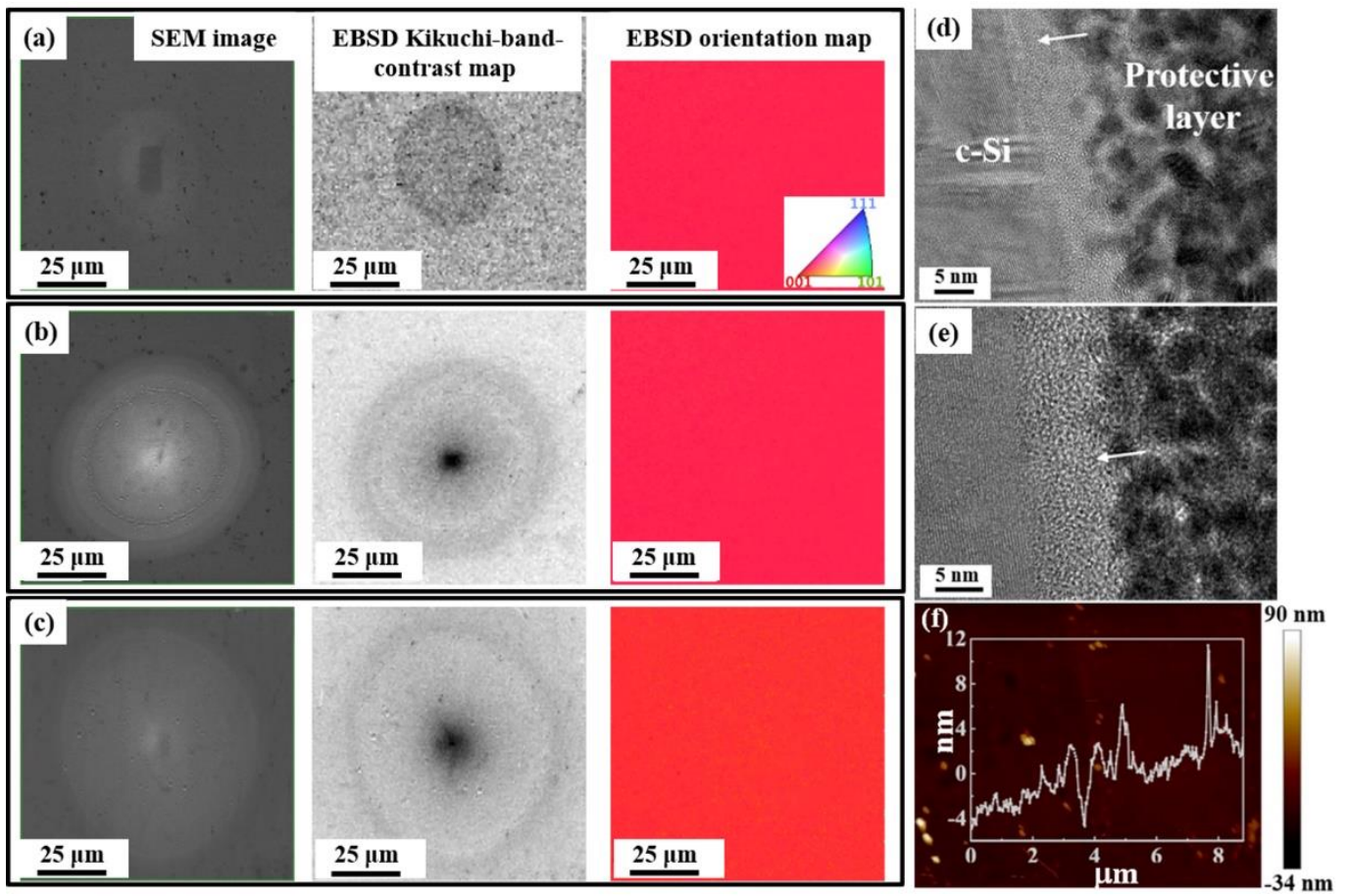

FIG. 4. SEM images and EBSD maps showing structural response of single-crystalline Si with the (100) surface to femtosecond laser peak-fluence of $0.29 \mathrm{~J} / \mathrm{cm}^{2}$ (a), $0.57 \mathrm{~J} / \mathrm{cm}^{2}$ (b), and $1.43 \mathrm{~J} / \mathrm{cm}^{2}$ (c). TEM observation of the microstructure $\left(0.57 \mathrm{~J} / \mathrm{cm}^{2}\right)$ at the spot center $(\mathrm{d})$ and the spot edge (e). AFM image of the transition region between Zone $\mathrm{C}$ and Zone $\mathrm{D}$, and the radial surface profile of the same region $\left(0.57 \mathrm{~J} / \mathrm{cm}^{2}\right)(\mathrm{f})$. Note: in all the EBSD crystallographic orientation maps, regions are colored according to their crystallographic orientations and the color code triangle is given in the bottom right corner of (a).

The origin of the observed microstructural changes could be two-fold, mechanical and/or thermal. For evaluation of the residual strain produced by femtosecond laser irradiation, the EBSD data taken from the $<111>$ orientation is arranged as kernel-average-misorientation (KAM) map, as shown in Figs. 5(a) and (b). The KAM quantifies the average misorientation around a pixel in EBSD map with respect to the nearest neighbor pixels and often serves as a measure of local strain. From Fig. 4(b), it is seen that the crystallographic orientation gradient produced by femtosecond laser does not exceed $0.1 \%$ m. By using the equation proposed by Kubin et al $[19,20]$, the underlying dislocation density was estimated as $\rho \sim 2 \theta / x b$, where $\theta / x$ is the crystallographic orientation gradient (in radians)

and $b$ is the Burgers vector. According to $b=\frac{1}{2} \sqrt{2} a$ for face-centered cubic lattice structure, where $a$ is the lattice constant $(5.43 \AA$ for $\mathrm{Si}), b$ is calculated to be $0.38 \mathrm{~nm}$. The dislocation density $\rho$ is estimated to be $10^{13} \mathrm{~m}^{-2}$. From the dislocation density, the corresponding accumulated shear strain $\gamma$ is evaluated by $\gamma \sim \rho b L$ [21], where $L$ is a slip distance. Assuming the slip distance to be $10 \mu \mathrm{m}$ (i.e. a characteristic scale of the crystallographic orientation gradients in Fig. 5(b)), the laser-induced strain is on the order of 0.01 . Therefore, the effect of the laser induced strain should be relatively low. 

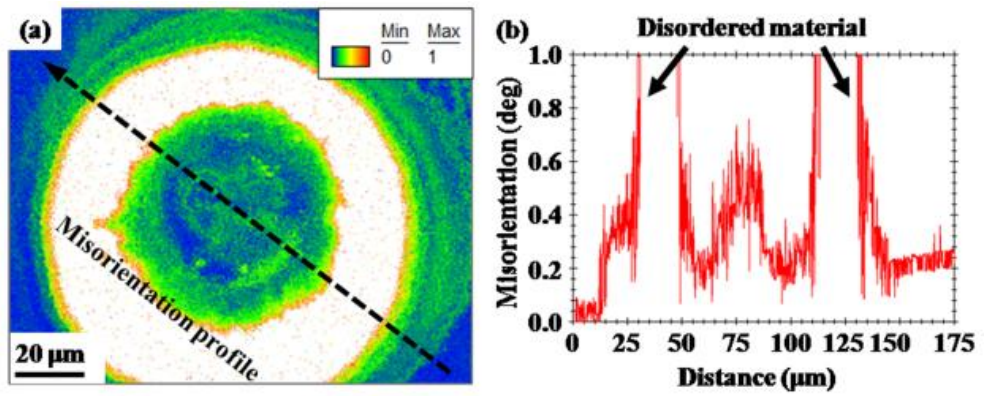

FIG. 5. EBSD kernel-average-misorientation (KAM) map (a), and EBSD KAM profile (b) of the laser irradiated spot produced on the (111) surface. Note: The data were taken from polycrystalline Si irradiated at laser peak-fluence of $0.99 \mathrm{~J} / \mathrm{cm}^{2}$.

The thermal effects during ultrafast laser irradiation was evaluated by the two-temperature model (TTM), which has been widely used for studying this scenario [22-27]. For semiconductors, three coupled equations are used to describe the dynamics of carrier density, carrier temperature, and lattice temperature, respectively [26, 27]. The change of carrier density is accompanied with the change of the energy stored in carriers. In addition, carriers first absorb photon energy to reach a high temperature due to small heat capacity and lattice acquires energy through carrier-lattice scattering until carriers and lattice reach thermal equilibrium.

$$
\begin{gathered}
\frac{\partial n}{\partial t}=\frac{\alpha I(x, t)}{h v}+\frac{\beta I^{2}(x, t)}{2 h v}-\gamma n^{3}+\theta n-\nabla \cdot \mathbf{u} \\
C_{e-h} \frac{\partial T_{e}}{\partial t}=(\alpha+\Theta n) I(x, t)+\beta I^{2}(x, t)-\nabla \cdot W-\frac{C_{e-h}}{\tau_{e}}\left(T_{e}-T_{l}\right) \\
-\frac{\partial n}{\partial t}\left(E_{g}+3 k_{B} T_{e}\right)-n\left(\frac{\partial E_{g}}{\partial T_{l}} \frac{\partial T_{l}}{\partial t}+\frac{\partial E_{g}}{\partial n} \frac{\partial n}{\partial t}\right) \\
C_{l} \frac{\partial T_{l}}{\partial t}=\nabla \cdot\left(k_{l} \nabla T_{l}\right)+\frac{C_{e-h}}{\tau_{e}}\left(T_{e}-T_{l}\right)
\end{gathered}
$$

Equation (1) describes the dynamics of carrier density. The five terms on the right-hand side of Eq. (1) account for one-photon absorption, two-photon absorption, Auger combination, impact ionization, and spatial diffusion, respectively. In this work, the laser photon energy $(1.2 \mathrm{eV})$ is slightly larger than the band gap of $\mathrm{Si}(1.12 \mathrm{eV})$, so it is reasonable to consider one- and two-photon absorption while higher-order absorption, which is less possible, can be neglected. The carrier pair current is evaluated as

$$
\text { ư }=-D_{0}\left(\nabla n+\frac{n}{2 k_{B} T_{e}} \nabla E_{g}+\frac{n}{2 T_{e}} \nabla T_{e}\right)
$$

Equation (2) describes the evolution of the carrier temperature. The first two terms on the right-hand side of Eq. (2) denote laser heating due to one-photon and two-photon absorption, respectively, where $\Theta n$ describes absorption of light by free carriers created by interband transition. The third term indicates the rate of energy loss due to the ambipolar energy current

$$
\stackrel{\text { um }}{W}=\left(E_{g}+4 k_{B} T_{e}\right) \stackrel{\text { ü }}{J}-\left(k_{e}+k_{h}\right) \nabla T_{e}
$$

The fourth term accounts for the thermal coupling between carriers and lattice, which cools down carriers and heats up lattice. The last two terms represent energy loss or generation rate in electronhole pairs, induced by the change of carrier density. Equation (3) models the temperature change of 
lattice. The two terms on the right-hand side of the equation indicate heat transfer due to thermal diffusion within the lattice and the coupling between carriers and lattice. The attenuation of laser intensity $I(x, t)$ in the propagation direction is obtained by considering the loss due to one-photon and two-photon absorption:

$$
\frac{\partial I}{\partial x}=-(\alpha+\Theta n) I-\beta I^{2}
$$

After integration, the volumetric heating by the laser is modeled as:

$$
I(x, t)=\frac{(\alpha+\Theta n) I_{0} \exp [-(\alpha+\Theta n) x]}{(\alpha+\Theta n)+\beta I_{0}[1-\exp (1-(\alpha+\Theta n) x)]}
$$

where $I_{0}$ is the laser intensity at the surface of the sample $(x=0)$ evaluated as:

$$
I_{0}(t)=\sqrt{\frac{4 \ln 2}{\pi}} \frac{(1-R) \phi}{t_{p}} \exp \left[-4 \ln 2\left(\frac{t-3 t_{p}}{t_{p}}\right)^{2}\right]
$$

where $R$ denotes the surface reflectivity, $\phi$ the pulse fluence, and $t_{\mathrm{p}}$ the full width at half maximum

\begin{tabular}{|c|c|c|c|}
\hline Parameters & Nomenclature & Values & Reference \\
\hline$\lambda(\mathrm{nm})$ & Wavelength & 1030 & \\
\hline$t_{p}(\mathrm{fs})$ & Pulse width & 800 & \\
\hline$\phi\left(\mathrm{J} / \mathrm{cm}^{2}\right)$ & Pulse fluence & 0.47 & \\
\hline$k_{l}(\mathrm{~W} / \mathrm{m} \mathrm{K})$ & $\begin{array}{c}\text { Lattice thermal } \\
\text { conductivity }\end{array}$ & $1.585 \times 10^{5} T_{l}^{-1.23}$ & \multirow{11}{*}[24,26]{} \\
\hline$C_{l}\left(\mathrm{~J} / \mathrm{m}^{3}\right)$ & $\begin{array}{l}\text { Lattice specific } \\
\text { heat capacity }\end{array}$ & $1.978 \times 10^{6}+3.54 \times 10^{2} T_{l}-3.68 \times 10^{6} T_{l}^{-2}$ & \\
\hline $\begin{array}{c}k_{e}, k_{h}(\mathrm{eV} / \mathrm{s} \\
\mathrm{m} \mathrm{K})\end{array}$ & $\begin{array}{l}\text { Electron, hole } \\
\text { thermal } \\
\text { conductivity }\end{array}$ & $-3.47 \times 10^{18}+4.45 \times 10^{16} T_{\underline{e}}$ & \\
\hline$C_{e-h}\left(\mathrm{~J} / \mathrm{m}^{3}\right)$ & $\begin{array}{l}\text { Electron-hole } \\
\text { specific heat } \\
\text { capacity }\end{array}$ & $3 n k_{B}$ & \\
\hline$\tau_{e}(\mathrm{fs})$ & $\begin{array}{l}\text { Electron-lattice } \\
\text { coupling time }\end{array}$ & $240\left(1+n / 6 \times 10^{20}\right)$ & \\
\hline$\gamma\left(\mathrm{m}^{6} / \mathrm{s}\right)$ & Auger coefficient & $3.8 \times 10^{-43}$ & \\
\hline$\theta\left(\mathrm{s}^{-1}\right)$ & $\begin{array}{l}\text { Impact ionization } \\
\text { coefficient }\end{array}$ & $3.6 \times 10^{10} \exp \left(-1.5 E_{g} / k_{B} T_{e}\right)$ & \\
\hline$D_{0}\left(\mathrm{~m}^{2} / \mathrm{s}\right)$ & $\begin{array}{l}\text { Electron } \\
\text { diffusivity }\end{array}$ & $1.8 \times 10^{-3}\left(300 / T_{l}\right)$ & \\
\hline$E_{g}(\mathrm{eV})$ & Band gap & $1.16-7.02 \times 10^{-4} T_{l}^{-2} /\left(T_{l}+1108\right)-1.5 \times 10^{-8} n^{1 / 3}$ & \\
\hline$R$ & Reflectivity & $0.37+5 \times 10^{-5}\left(T_{l}-300\right)$ & \\
\hline$n_{0}\left(\mathrm{~m}^{-3}\right)$ & $\begin{array}{c}\text { Initial carrier } \\
\text { density }\end{array}$ & $10^{18}$ & \\
\hline
\end{tabular}
(FWHM) of the pulse. The nomenclature of this model is summarized in Table 1.

Table 1 The parameters used in the simulation. 


\begin{tabular}{|c|c|c|c|}
\hline$\alpha\left(\mathrm{m}^{-1}\right)$ & $\begin{array}{l}\text { One-photon } \\
\text { absorption } \\
\text { coefficient }\end{array}$ & $\begin{array}{c}-5895+62.62 T_{l}-0.2309 T_{l}^{2} \\
+3.186 \times 10^{-4} T_{l}^{3}+9.967 \times 10^{8} T_{l}^{4}-1.409 \times 10^{-11} T_{l}^{5}\end{array}$ & \multirow{3}{*}{ [27] } \\
\hline$\beta(\mathrm{m} / \mathrm{W})$ & $\begin{array}{l}\text { Two-photon } \\
\text { absorption } \\
\text { coefficient }\end{array}$ & $1.5 \times 10^{-11}$ & \\
\hline$\Theta\left(\mathrm{m}^{2}\right)$ & $\begin{array}{l}\text { Free carrier } \\
\text { absorption } \\
\text { coefficient }\end{array}$ & $5 \times 10^{-22}\left(T_{l} / 300\right)$ & \\
\hline
\end{tabular}

With this model, the peak lattice temperature and the carrier density versus the utilized laser fluence are calculated and presented in Fig. 6(a) and Fig. 6(b), respectively. At $0.29 \mathrm{~J} / \mathrm{cm}^{2}$, amorphization of Si happens as shown in Fig. 2(a) while the peak lattice temperature (Fig. 6(a)) is far below the melting temperature of $\mathrm{Si}(1687 \mathrm{~K})$ and the peak carrier density reaches about $4 \times 10^{26}$ $\mathrm{m}^{-3}$ (Fig. 6(b)). Due to the lack of data for the temperature-dependent reflectance $R$ at $1030 \mathrm{~nm}$, the reflectance in our calculation was cited from Ref. [26] for $775 \mathrm{~nm}$, which will slightly underestimate the carrier density since Si has lower reflectance at $1030 \mathrm{~nm}$. Considering this underestimation, the critical carrier density for structural change in our work agrees well with that the previously reported value $6.9 \times 10^{26} \mathrm{~m}^{-3}$ irradiated by $1030 \mathrm{~nm}$ laser, attributed to non-thermal phase change with high electron density and cold lattice [27]. For higher values of fluence used in this work, before being heated to the melting point, the lattice crushes when the electron density exceeds the critical value, which occurs earlier. Therefore, the structural change of Si with the femtosecond laser parameters in this work has the non-thermal nature.
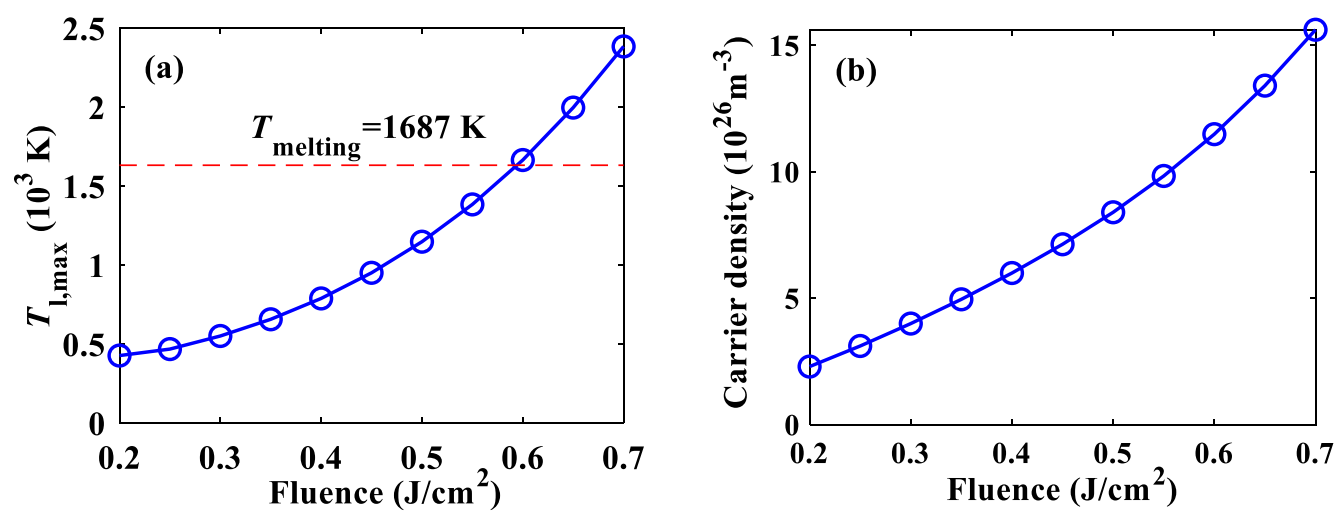

FIG. 6. Calculated relation between the peak lattice temperature and laser fluence (a) and calculated relation between the peak carrier density and laser fluence (b).

Generally, amorphization occurs at relatively high solidification rate. The preference of amorphization on the (111) surface could be qualitatively explained by the theorem of Wulff construction [28]: the growth speed of a crystallographic plane is proportional to its surface energy under equilibrium. Since Si has face-centered cubic lattice structure, the (111) surface is the crystallographic close-packed plane and has the smallest surface energy. Accordingly, the amorphization may be energetically favorable than the crystallization with the same solidification 
rate. While this dependence on crystallographic orientation is qualitatively similar to the case in nanosecond laser heating, there is a quantitative difference. In a previous work using nanosecond laser for Si processing, there is a range of fluence, for which amorphization can be induced on different crystallographic surfaces including the (100) surface [10]. However, in this work, no amorphization occurs on the (100) surface even for the lowest fluence $0.29 \mathrm{~J} / \mathrm{cm}^{2}$, below which structural modification is hardly generated (Fig. 4). This can be explained by the non-thermal melting mechanism caused by femtosecond laser, which induces mild heating, smaller thermal gradient, and slower cooling compared with nanosecond laser. Therefore, with non-thermal melting, recrystallization is favored. The formation of the ring-type amorphization region is related to the Gaussian distribution of laser intensity. According to a previous simulation study [29], the thermal gradient and the solidification interface velocity both decrease with increased laser intensity. In the central region of the laser spot, the intensity is high and the interface velocity is low, so that crystallization reforms after non-thermal melting. In the periphery of the laser spot, the intensity is low and crystal grains cannot grow in time due to high interface velocity, resulting in amorphization.

\section{Conclusion}

In this work, the crystallographic orientation dependence of amorphization effect of $\mathrm{Si}$ with femtosecond laser irradiation is studied. Extensive microstructural examinations reveal a formation of amorphized silicon within the laser irradiated surface. From two-temperature model simulations as well as experimental observations, it is deduced that the amorphous phase originates from nonthermal melting-to-recrystallization transformation sequence. A significant sensitivity of microstructural changes to crystallographic orientation of the laser irradiated surface is also found. It is revealed that surfaces of different crystallographic orientations have different tendencies to form amorphization. Within the laser fluence range in this work, the (111) surface of Si favors generation of amorphization compared with the other crystallographic planes. This phenomenon could be explained by the lowest crystallization speed required by the (111) surface due to its smallest surface energy. In addition, femtosecond laser-induced non-thermal melting induces mild thermal gradient and favors recrystallization in comparison with nanosecond laser-induced thermal melting.

\section{Acknowledgment}

This experimental work is supported by the National Key Research and Development Program of China (2017YFB1104803). This theoretical work is supported by the National Natural Science Foundation of Guangdong Province (2019A1515010745). The authors acknowledge assistance of Qing He in laser irradiation experiment.

\section{References}

[1] J. Jia, M. Li, C. V. Thompson, Amorphization of silicon by femtosecond laser pulses, Appl. Phys. Lett. 84 (2004) 3205-3207. https://doi.org/10.1063/1.1719280.

[2] Y. Izawa, Y. Setuhara, M. Hashida, M. Fujita, Y.Izawa, Ablation and Amorphization of Crystalline Si by Femtosecond and Picosecond Laser Irradiation, Jpn. J. Appl. Phys. 45 (2006) 5791. https://doi.org/10.1143/JJAP.45.5791.

[3] Y. Izawa, Y.Izawa, Y. Setsuhara, M. Hashida, R. Sasaki, H. Nagai, M. Yoshida, Ultrathin amorphous Si layer formation by femtosecond laser pulse irradiation. Appl. Phys. Lett. 90 (2007) 
044107. https://doi.org/10.1063/1.2431709.

[4] M. Rini, N. Dean, J. Itatani, Y. Tomioka, R. W. Schoenlein, A. Cavalleri, Control of the electronic phase of a manganite by mode-selective vibrational excitation, Nature. 449 (2007) 72-74. https://doi.org/10.1038/nature06119.

[5] S. Wall, D. Wegkamp, L. Foglia, K. Appavoo, J. Nag, R. F. Haglund, M. Wolf, Ultrafast changes in lattice symmetry probed by coherent phonons, Nat. Commun. 3 (2012) 1-6. https://doi.org/10.1038/ncomms1719.

[6] M. Hase, P. Fons, K. Mitrofanov, A. V. Kolobov, J. Tominaga, Femtosecond structural transformation of phase-change materials far from equilibrium monitored by coherent phonons, Nat. Commun. 6 (2015) 8367. https://doi.org/10.1038/ncomms9367.

[7] T. H. R. Crawford, G. A. Botton, H. K. Haugen, Crystalline orientation effects on conical structure formation in femtosecond laser irradiation of silicon and germanium, Appl. Surf. Sci. 256 (2010) 1749-1755. https://doi.org/10.1016/j.apsusc.2009.09.107.

[8] X. Sedao, C. Maurice, F. Garrelie, J. P. Colombier, S. Reynaud, R. Quey, F. Pigeon, Influence of crystal orientation on the formation of femtosecond laser-induced periodic surface structures and lattice defects accumulation, Appl. Phys. Lett. $104 \quad$ (2014) 171605. https://doi.org/10.1063/1.4874626.

[9] J. M. Liu, Simple technique for measurements of pulsed Gaussian-beam spot sizes, Opt. Lett. 7 (1982) 196-198. https://doi.org/10.1364/OL.7.000196.

[10] A. G. Cullis, N. G. Chew, H. C. Webber, D. J. Smith, Orientation dependence of high speed silicon crystal growth from the melt, J. Cryst. Growth. 68 (1984) 624-638. https://doi.org/10.1016/0022-0248(84)90469-X.

[11] J. A. Yater, M. O. Thompson, Orientation dependence of laser amorphization of crystal Si, Phys. Rev. Lett. 63 (1989) 2088. https://doi.org/10.1103/PhysRevLett.63.2088.

[12] S. K. Sundaram, E. Mazur, Inducing and probing non-thermal transitions in semiconductors using femtosecond laser pulses, Nat. Mater. 1 (2002) 217-224. https://doi.org/10.1038/nmat767.

[13] M. Z. Mo, Z. Chen, R. K. Li, M. Dunning, B. B. L. Witte, J. K. Baldwin, A. H. Reid, Heterogeneous to homogeneous melting transition visualized with ultrafast electron diffraction, Science. 360 (2018) 1451-1455. https://doi.org/10.1126/science.aar2058.

[14] L. Jiang, A. D. Wang, B. Li, T. H. Cui, Y. F. Lu, Electrons dynamics control by shaping femtosecond laser pulses in micro/nanofabrication: modeling, method, measurement and application, Light-Sci. Appl. 7 (2018) 17134-17134. https://doi.org/10.1038/1sa.2017.134.

[15] J. Bonse, K.W. Brzezinka, A.J. Meixner, Modifying single-crystalline silicon by femtosecond laser pulses: an analysis by micro Raman spectroscopy, scanning laser microscopy and atomic force microscopy, Appl. Surf. Sci. 221(2004) 215-230. https://doi.org/10.1016/S0169-4332(03)00881-X. [16] J. Bonse, All-optical characterization of single femtosecond laser-pulse-induced amorphization in silicon, Appl. Phys. A: Mater. Sci. Process. A84 (2006) 63-66. https://doi.org/10.1007/s00339006-3583-3.

[17] Y. Fuentes-Edfuf, M. Garcia-Lechuga, D. Puerto, C. Florian, A. Garcia-Leis, S. Sanchez-Cortes, Fabrication of amorphous micro-ring arrays in crystalline silicon using ultrashort laser pulses, Appl. Phys. Lett. 110(2017) 211602. https://doi.org/10.1063/1.4984110.

[18] Y. Izawa, Y. Setuhara, M. Hashida, M. Fujita, Y. Izawa, Ablation and amorphization of crystalline Si by femtosecond and picosecond laser irradiation, Jpn. J. Appl. Phys. 45 (2006) 5791. https://doi.org/10.1143/JJAP.45.5791. 
[19] L. P. Kubin, A. Mortensen, Geometrically necessary dislocations and strain-gradient plasticity: a few critical issues, Scr. Mater. 48 (2003) 119-125. https://doi.org/10.1016/S1359-6462(02)003354.

[20] M. Calcagnotto, D. Ponge, E. Demir, D. Raabe, Orientation gradients and geometrically necessary dislocations in ultrafine grained dual-phase steels studied by 2D and 3D EBSD, Mater. Sci. Eng: A. 527 (2010) 2738-2746. https://doi.org/10.1016/j.msea.2010.01.004.

[21] H. Mecking, K. Lücke, A new aspects of the theory of flow stress of metals, Scripta Metall. 4 (1970) 427-432. https://doi.org/10.1016/0036-9748(70)90078-5.

[22] M. I. Kaganov, I. M. Lifshitz, L. V. Tanatarov, Relaxation between electrons and the crystalline lattice, Sov. Phys. JETP 4 (1957) 173-178.

[23] S. I. Anisimov, B. L. Kapeliovich, T. L. Perel'man, Electron emission from metal surfaces exposed to ultrashort laser pulses, Sov. Phys. JETP 39 (1974) 375-377.

[24] H. M. van Driel, Kinetics of high-density plasmas generated in Si by 1.06- and 0.53-pm picosecond laser pulses, Phys. Rev. B $35 \quad$ (1987) 8166-8176. https://doi.org/10.1103/PhysRevB.35.8166.

[25] M. I. Gallant, H. M. van Driel, Infrared reflectivity probing of thermal and spatial properties of laser-generated carriers in germanium, Phys. Rev. B 26 (1982) 2133-2146. https://doi.org/10.1103/PhysRevB.26.2133.

[26] J. K. Chen, D. Y. Tzou, J. E. Beraun, Numerical investigation of ultrashort laser damage in semiconductors, Int. J. Heat Mass Transfer. 48 (2005) 501-509. https://doi.org/10.1016/j.ijheatmasstransfer.2004.09.015.

[27] T. Jostein, E. F. Sean, Temperature dependent ablation threshold in silicon using ultrashort laser pulses, J. Appl. Phys. 112 (2012) 103514. https://doi.org/10.1063/1.4766380.

[28] R. Vanselow, R. Howe, Chemistry and Physics of Solid Surfaces VII, Berlin, Heidelberg : Springer Berlin Heidelberg, 1988.

[29] A. G. Cullis, H. C. Webber, N. G. Chew, J. M. Poate, P. Baeri, Transitions to defective crystal and the amorphous state induced in elemental Si by laser quenching, Phys. Rev. Lett. 49 (1982) 219. https://doi.org/10.1103/PhysRevLett.49.219. 
Figures
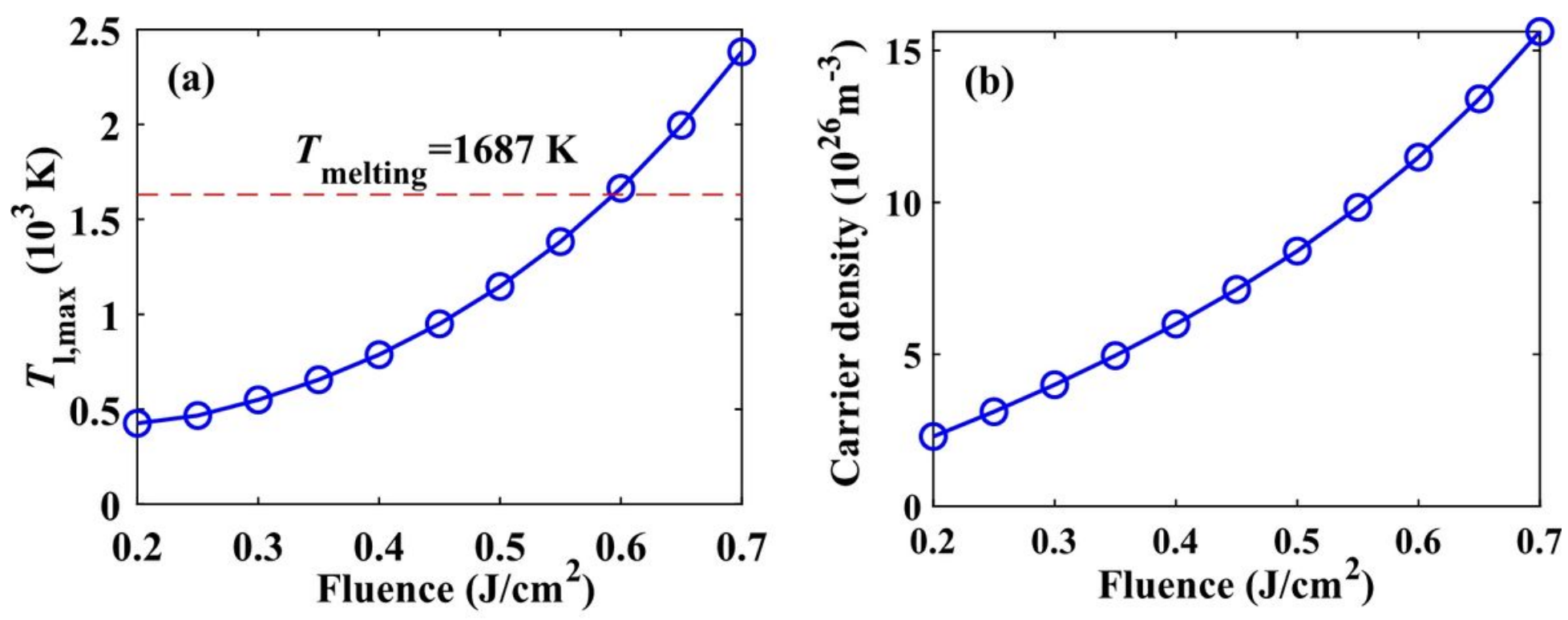

Figure 1

SEM image (a), EBSD Kikuchi-band-contrast map (b), and EBSD crystallographic orientation map (c) showing anisotropic structural response to femtosecond laser irradiation. In (b), the inset is the magnified view of the laser-induced spot in the (111) surface. In (c), individual grains are colored according to their crystallographic orientation and the color code triangle is shown in the top right corner. Note: The data are taken from polycrystalline Si irradiated at laser peak-fluence of $0.99 \mathrm{~J} / \mathrm{cm} 2$.
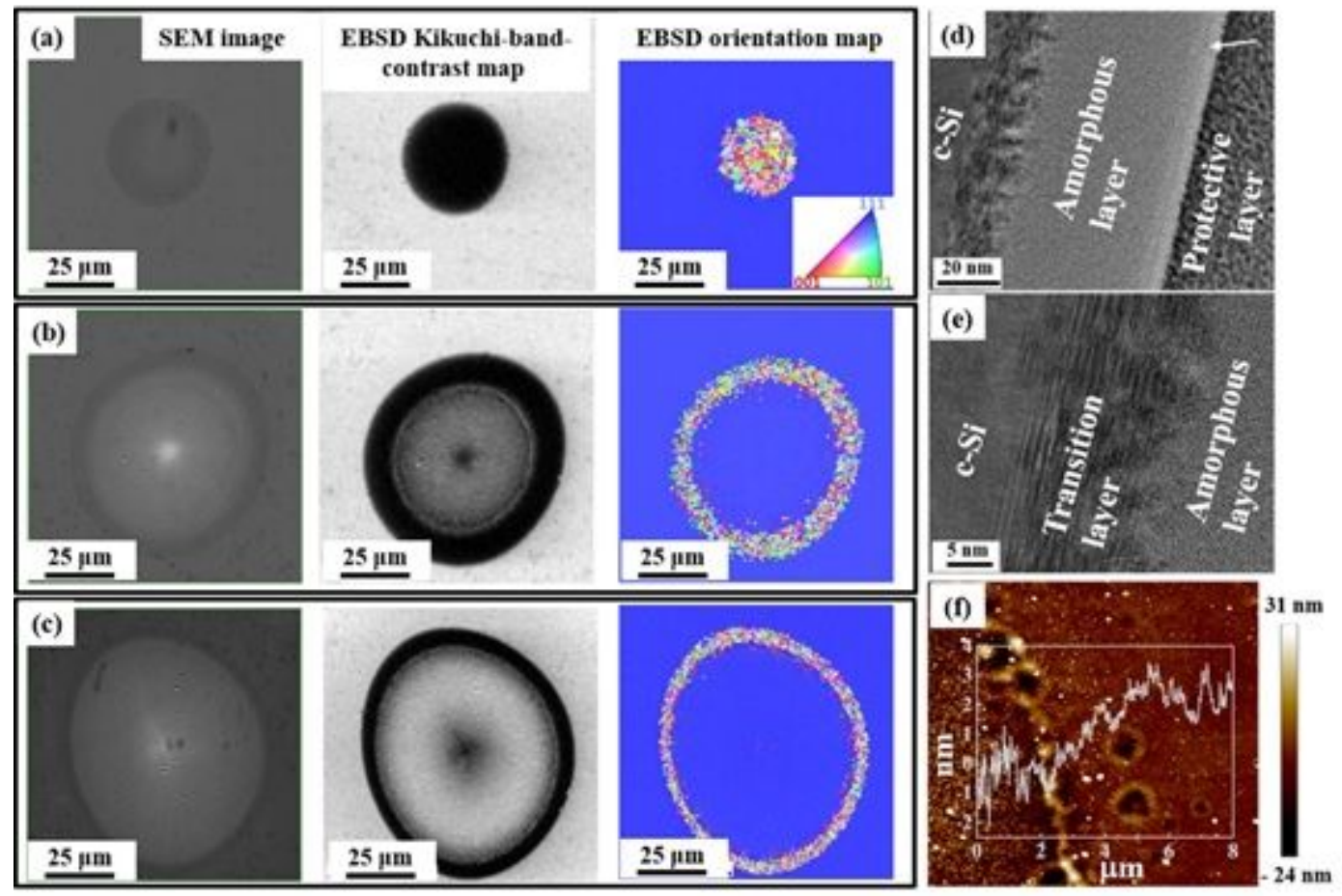

Figure 2 
SEM images and EBSD maps showing structural response of single-crystalline Si with the (111) surface to femtosecond laser peak-fluence of $0.29 \mathrm{~J} / \mathrm{cm} 2$ (a), $0.57 \mathrm{~J} / \mathrm{cm} 2$ (b), and $1.43 \mathrm{~J} / \mathrm{cm} 2$ (c). TEM observation of the microstructure $(0.57 \mathrm{~J} / \mathrm{cm} 2)$ in the amorphous-crystalline transition layer (zone $\mathrm{D})$ : low magnification (d) and high magnification (e). AFM image of the transition region between Zone $C$ and Zone $D$, and the radial surface profile of the same region $(0.57 \mathrm{~J} / \mathrm{cm} 2)(\mathrm{f})$. Note: in all the EBSD crystallographic orientation maps, regions are colored according to their crystallographic orientations and the color code triangle is given in the bottom right corner of (a).

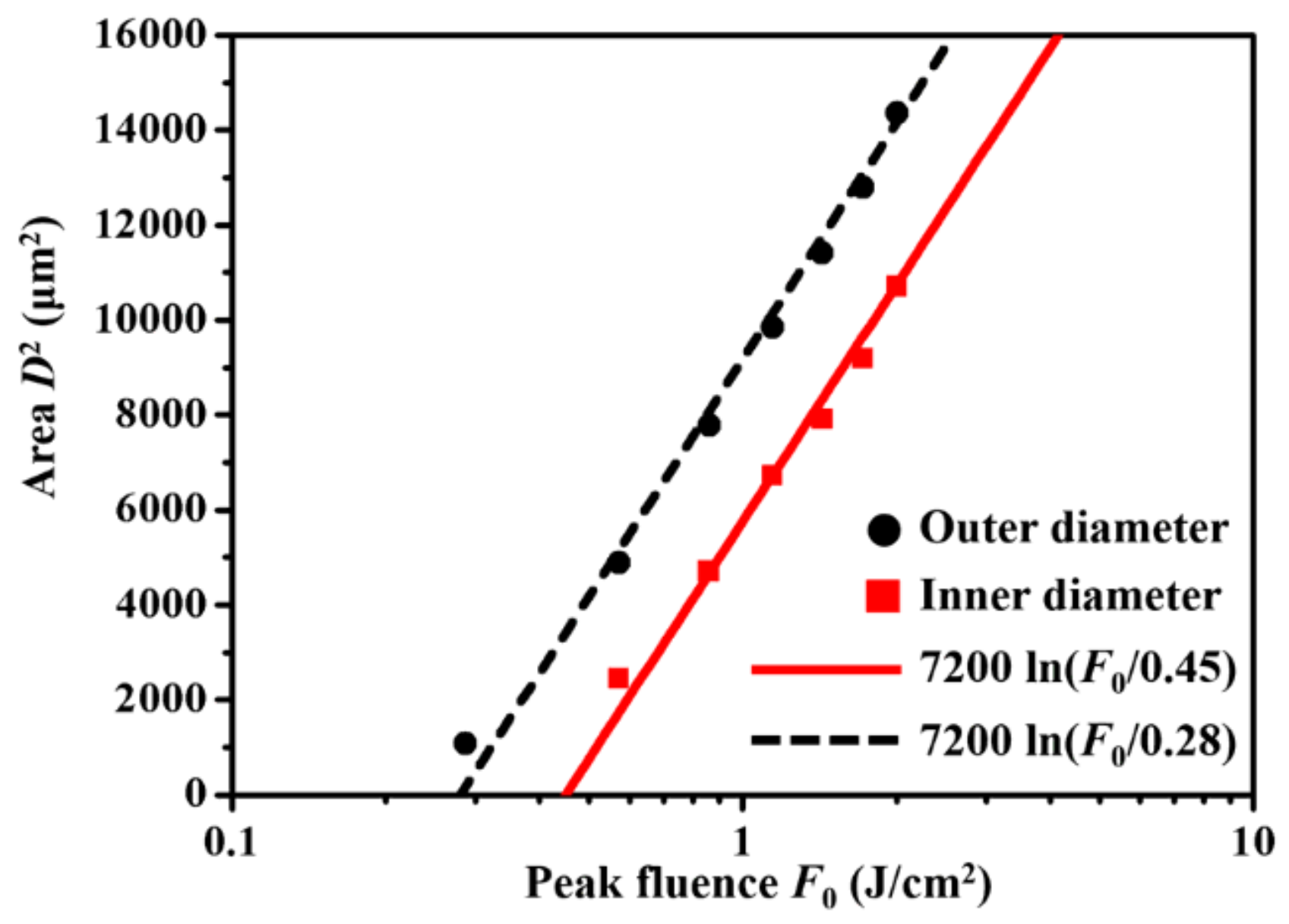

Figure 3

Dependence of ablation area (Zone C) and amorphization area (Zone D) on laser peak-fluence. Note: The data were taken from single-crystalline Si with the (111) surface. 

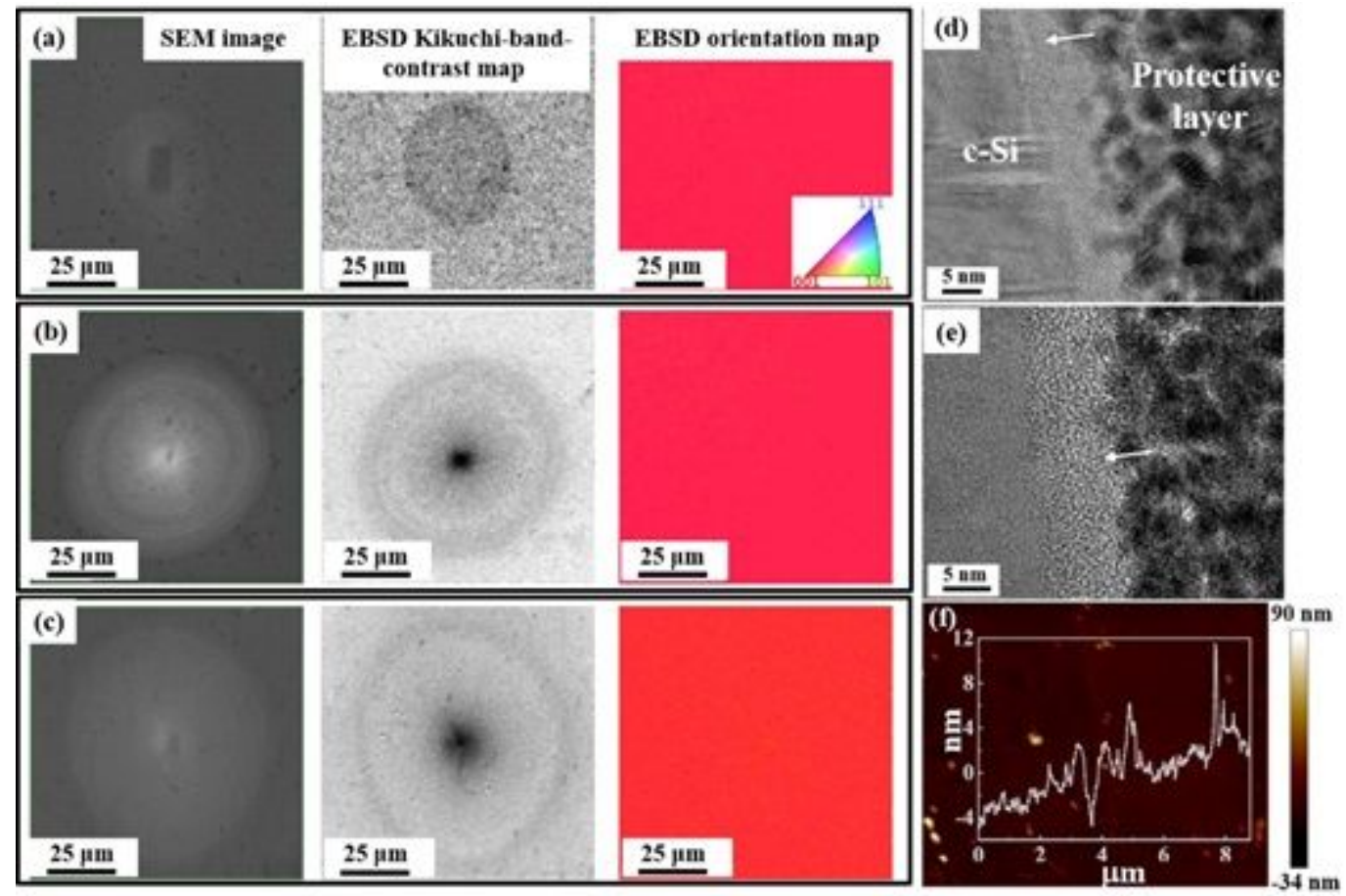

Figure 4

SEM images and EBSD maps showing structural response of single-crystalline Si with the (100) surface to femtosecond laser peak-fluence of $0.29 \mathrm{~J} / \mathrm{cm} 2(a), 0.57 \mathrm{~J} / \mathrm{cm} 2(\mathrm{~b})$, and $1.43 \mathrm{~J} / \mathrm{cm} 2$ (c). TEM observation of the microstructure $(0.57 \mathrm{~J} / \mathrm{cm} 2)$ at the spot center (d) and the spot edge (e). AFM image of the transition region between Zone $C$ and Zone $D$, and the radial surface profile of the same region ( 0.57 $\mathrm{J} / \mathrm{cm} 2$ ) (f). Note: in all the EBSD crystallographic orientation maps, regions are colored according to their crystallographic orientations and the color code triangle is given in the bottom right corner of (a).
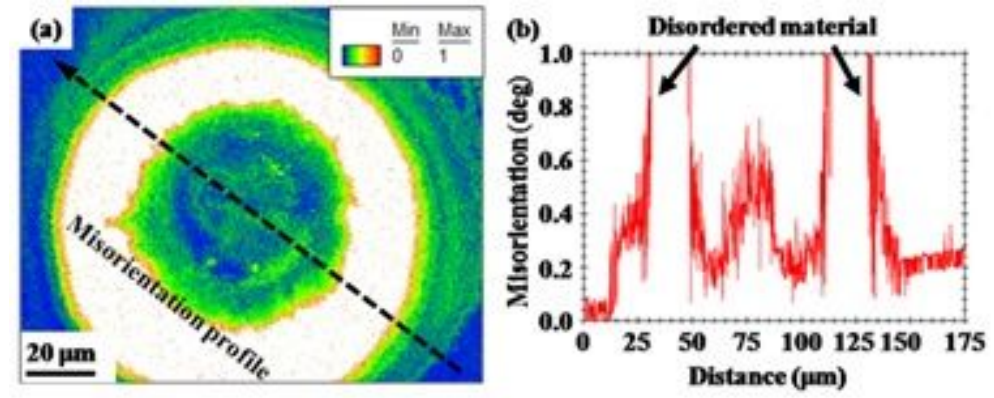

Figure 5

EBSD kernel-average-misorientation (KAM) map (a), and EBSD KAM profile (b) of the laser irradiated spot produced on the (111) surface. Note: The data were taken from polycrystalline Si irradiated at laser peakfluence of $0.99 \mathrm{~J} / \mathrm{cm} 2$. 

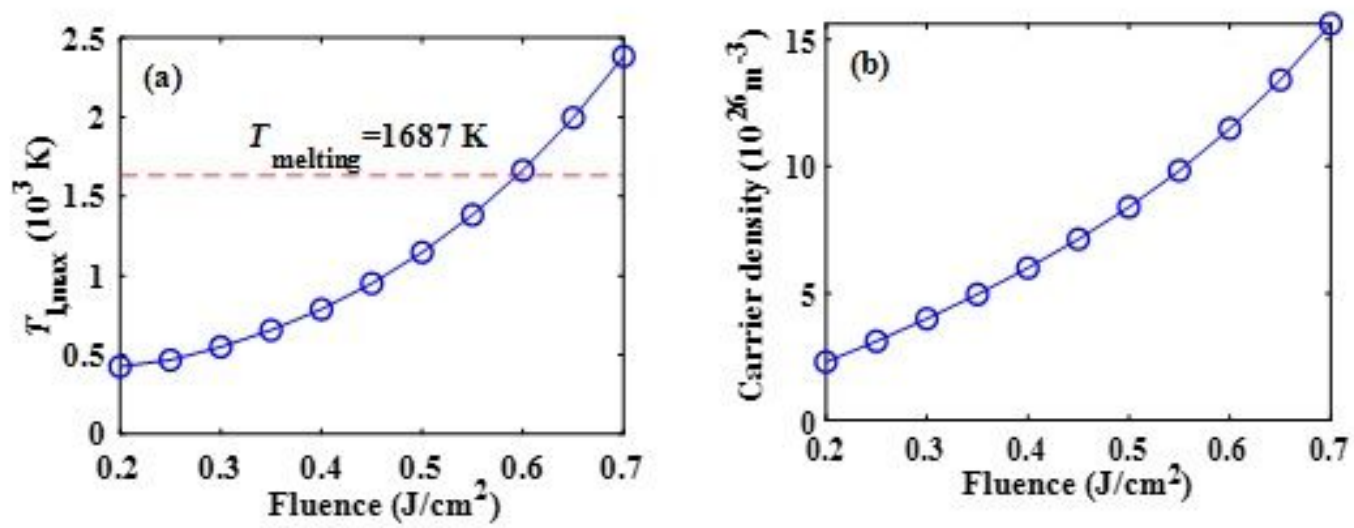

Figure 6

Calculated relation between the peak lattice temperature and laser fluence (a) and calculated relation between the peak carrier density and laser fluence (b). 